\title{
Cerebral atrophy in myotonic dystrophy: a voxel based morphometric study
}

\author{
G Antonini, C Mainero, A Romano, F Giubilei, V Ceschin, F Gragnani, S Morino, M Fiorelli, \\ F Soscia, A Di Pasquale, F Caramia
}

J Neurol Neurosurg Psychiatry 2004;75:161 1-1613. doi: 10.1136/jnnp.2003.032417

\begin{abstract}
Brain involvement in myotonic dystrophy type 1 (DM1) is characterised by cortical atrophy and white matter lesions. We compared the magnetic resonance imaging derived grey matter maps of 22 DM1 patients with those of matched, healthy controls using voxel based morphometry to evaluate the extension of global and regional cortical atrophy in DMI, as well as its relationships with clinical and genetic features. Patients had significantly reduced brain tissue volumes. Grey matter volume was inversely correlated with age; this inverse correlation was significantly stronger in DMl than in controls. Neither the clinical and genetic characteristics nor white matter lesions were correlated with cortical atrophy. Grey matter atrophy was located mainly in the bilateral frontal and parietal lobes, in the bilateral middle temporal gyrus, and in the left superior temporal and occipital gyrus.
\end{abstract}

M yotonic dystrophy type 1 (DMl) is one of the most common inherited neuromuscular disorders in humans. ${ }^{1}$ It is a progressive autosomal dominant, multisystemic disease, characterised by an unstable triplet cytosine thymine guanidine (CTG) repeat on chromosome 19, which appears to be excessively amplified.

In addition to neuromuscular symptoms, many DMl patients develop intellectual impairment, hypersomnia, and affective disorders. Imaging studies have documented brain involvement consisting of hypoperfusion, hypometabolism, white matter lesions, ventricular dilatation, and global brain atrophy. ${ }^{2-9}$

Voxel based morphometry (VBM) can be used to evaluate both global and regional changes in brain volumes. ${ }^{10}$ In this study we used VBM to map cortical and subcortical grey matter atrophy in DMl.

\section{METHODS \\ Subjects}

A total of 22 consecutive DMl patients ( 13 males and nine females; median age 33 years, range 20-55 years) and 22 sex and age matched healthy subjects were included in the study. Patients with congenital DMl or any other neurological disease which might affect muscle or cognitive function were excluded. Disease duration ranged from 1 to 35 years (median: 12.5 years). Age at onset of disease ranged from 2 to 47 years (median: 19 years). Disease onset in five patients occurred before 14 years of age. The patients' educational level ranged from 8 to 17 years (median: 11 years). CTG triplet expansion, evaluated in all the subjects from leukocytes, ranged from 96 to 1570, mean (SD) 539 (365). The muscular disability of the patients measured by means of a muscular disability rating scale divided into five grades, ${ }^{11}$ was grade I (no clinical muscular impairment) in four patients, II (minimal signs: myotonia, facial weakness) in nine, III (mild muscle weakness: no external help is required in everyday tasks) in five, and IV (moderate muscle weakness: external help is required in everyday tasks) in four. Seven patients complained of hypersomnia (Epworth Scale score higher than 10). The psychiatric examination, consisting of a semistructured interview designed to assess affective and personality abnormalities, showed that none of the patients met the DSM-IV (Diagnostic and statistical manual of mental disorders, fourth edition) criteria for a major disorder in axis I while only one subject had an avoidant personality disorder in axis II. Apathy ( 5 points), avoidant ( 5 points), and passive-aggressive ( 3 points) personality traits emerged as the prevalent behavioural abnormalities. Feelings of inadequacy and fear of being negatively judged in social occasions and at work were the principal avoidant criteria, while criticism and oppositive attitude were the prevalent features of passive-aggressive traits. Rating scales (Hamilton Rating Scale for Depression, Hamilton Rating Scale for Anxiety, State-Trait Anxiety Inventory-Form Y) showed trait anxiety and high levels of state anxiety in nine patients, and moderate and mild depressive symptoms, respectively, in one and 10 patients.

\section{Magnetic resonance imaging scanning protocol}

Magnetic resonance imaging (MRI) was performed on a $1.5 \mathrm{~T}$ Magnet (Philips Gyroscan NT-2000). Axial Tl weighted spin echo images (TR/TE/NEX: 600/15/2, matrix: $256 \times 256$, FOV: $230 \mathrm{~mm}$, 40 slices $4 \mathrm{~mm}$ thick, gap 0 ) and proton density, T2 weighted fast spin echo images (TR/TE/NEX: 2500/24-90/2, matrix: $128 \times 256$, FOV: $230 \mathrm{~mm}$, 40 slices $4 \mathrm{~mm}$ thick, gap 0 ) were acquired from all subjects.

Total lesion load (LL) was calculated for T2 hyperintense lesions using a semi-automated local threshold contouring technique (DispImage, D Plummer, UCL Hospitals Trust, London, UK).

Volumetric analyses were performed on the Tl weighted images using SPM99 (Wellcome Department of Imaging Neuroscience, Institute of Neurology, University College London, UK, http://www.fil.ion.ucl.ac.uk/spm/) and the VBM technique. ${ }^{10}$ Firstly, an automated segmentation based on a combination of intensity driven and Bayesian classification was performed on the original structural Tl images, which were partitioned into grey matter (GM), white matter (WM), and cerebrospinal fluid (CSF). The segmented GM images were then subjected to an affine, non-linear spatial normalisation into standard Talairach and Tournoux space, using the standard GM SPM99 template. The normalised whole brain structural images were then segmented into GM, WM, CSF, and non-CSF partitions and subjected to a second

Abbreviations: DM1, myotonic dystrophy type 1; GM, grey matter, GMV, grey matter volume; LL, lesion load; TIV, total intracranial volume; VBM, voxel based morphometry; WM, white matter; WMV, white matter volume 
Table 1 Between group analysis (two sample $t$ test, SPM99): regions of relative accelerated loss of grey matter volume in patients $v$ controls (modulated data)

\begin{tabular}{|c|c|c|c|c|c|c|}
\hline \multicolumn{3}{|c|}{ Talairach coordinates } & \multicolumn{4}{|l|}{ Region } \\
\hline $\mathbf{x}$ & $y$ & $\mathbf{z}$ & Hemisphere & Anatomic & Brodman area & Z statistic \\
\hline-27 & -60 & 63 & $\mathrm{~L}$ & Superior parietal lobule & BA 7 & 5.54 \\
\hline-43 & 2 & 38 & $\mathrm{~L}$ & Middle frontal gyrus & BA 9 & 5.38 \\
\hline-31 & -65 & 58 & $\mathrm{~L}$ & Superior parietal lobule & BA 7 & 5.20 \\
\hline-65 & -52 & 8 & $\mathrm{R}$ & Middle temporal gyrus & BA 39 & 5.13 \\
\hline 19 & -15 & 72 & $\mathrm{R}$ & Superior frontal gyrus & BA 6 & 5.10 \\
\hline 47 & -76 & 25 & $\mathrm{~L}$ & Superior temporal gyrus & BA 22 & 5.10 \\
\hline 31 & -61 & 60 & $\mathrm{R}$ & Superior parietal lobule/precuneus & BA 7 & 5.05 \\
\hline 27 & -9 & 52 & $\mathrm{R}$ & Precentral gyrus & BA 4 & 4.94 \\
\hline-67 & -10 & 26 & L & Postcentral gyrus & BA 1-3 & 4.89 \\
\hline-2 & 14 & 8 & $\mathrm{~L}$ & Caudate & - & 4.88 \\
\hline 49 & -12 & 28 & $\mathrm{R}$ & Precentral gyrus & BA 4 & 4.87 \\
\hline 3 & 5 & 69 & $\mathrm{R}$ & Superior frontal gyrus & BA 6 & 4.81 \\
\hline-47 & 23 & 35 & $\mathrm{~L}$ & Middle frontal gyrus & BA 9 & 4.78 \\
\hline-69 & -37 & 20 & $\mathrm{~L}$ & Middle temporal gyrus & BA 21 & 4.77 \\
\hline 19 & -79 & 48 & $\mathrm{R}$ & Superior parietal lobule/precuneus & BA 7 & 4.76 \\
\hline 27 & -81 & 35 & $\mathrm{R}$ & Superior parietal lobule/precuneus & BA 7 & 4.65 \\
\hline 49 & 2 & 31 & $\mathrm{R}$ & Inferior frontal gyrus & BA 4 & 4.63 \\
\hline-33 & -66 & 36 & $L$ & Superior occipital gyrus & BA 19 & 4.61 \\
\hline 55 & -6 & 22 & $\mathrm{R}$ & Precentral gyrus & BA 4 & 4.60 \\
\hline-33 & -59 & 43 & $\mathrm{~L}$ & Inferior parietal lobule & BA 40 & 4.56 \\
\hline-33 & -69 & 45 & $\mathrm{R}$ & Superior parietal lobule & BA 7 & 4.48 \\
\hline
\end{tabular}

$B A$, Brodman area; L, left; $R$, right; $Z$, maximum $\zeta$ value (voxel level uncorrected $p<0.001$, cluster level corrected $p<0.05$ ).

extraction of normalised segmented GM and WM images so as to eliminate non-brain voxels. In order to preserve the GM volume within a voxel, voxel values in the segmented images were modulated using the Jacobian determinants derived from the spatial normalisation step. The modulated GM

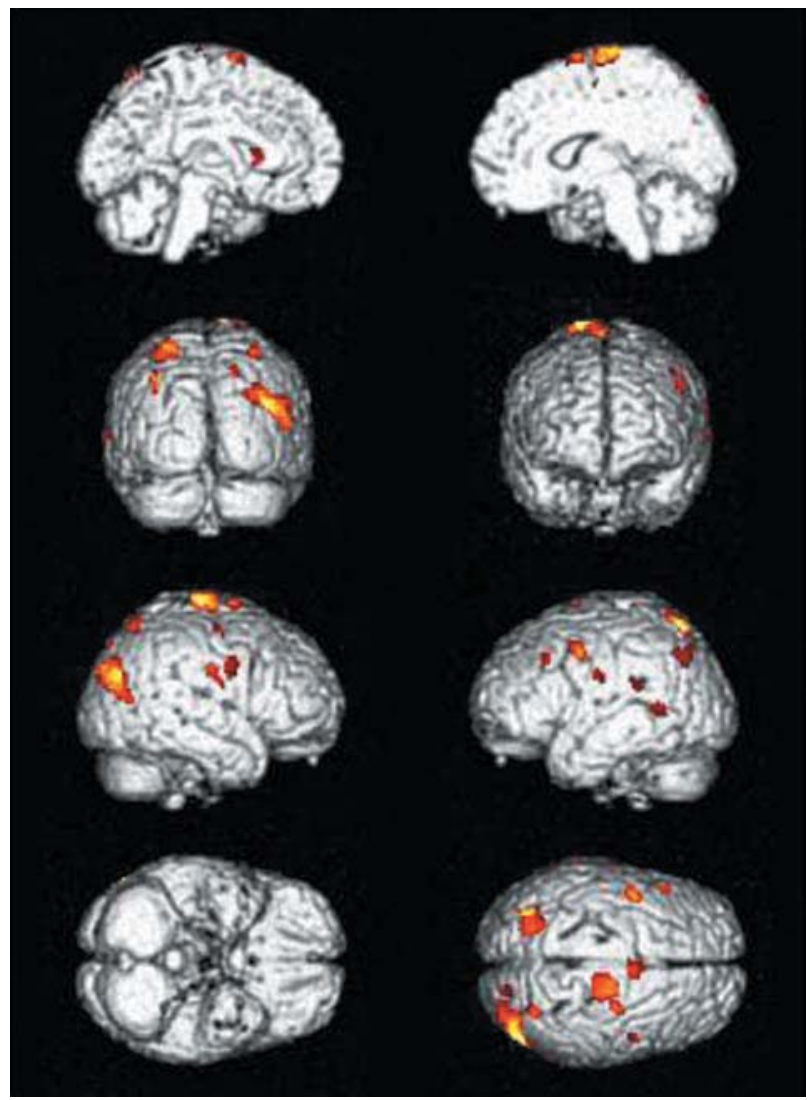

Figure 1 Regions of relative accelerated loss of grey matter volume in DM1 patients $v$ controls (between group analysis, SPM-99, two sample $t$ test, modulated data). images were then smoothed using a $12 \mathrm{~mm}$ FWHM (full width at half maximum) kernel.

Brain tissue volumes were calculated from the first set of segmented images using SPM99. We calculated the total intracranial volume (TIV) by adding together grey matter volume (GMV), white matter volume (WMV), and CSF volume.

Differences in TIV, GMV, and WMV between patients and controls were assessed by the two sample $t$ test, while the relationship with demographic, clinical, genetic, and conventional MR data was evaluated by linear regression analysis (SPSS 11.0).

Regional differences in GMV between DMl patients and controls were assessed using the smoothed modulated GM images and SPM99 ${ }^{12}$ (random effect analysis, two sample $t$ test). Clusters of voxels which had a peak $\mathrm{Z}$ score $>3.1$ (amplitude threshold uncorrected $\mathrm{p}<0.001$, extent threshold corrected $\mathrm{p}<0.05$ ) were considered significantly different.

Regional differences in WMV between DMI patients and controls were not assessed since VBM relies on the proportion of tissue under investigation in a voxel after smoothing. It is most suited for voxels in which this proportion is around $50 \%$. As white matter is found in large solid regions, much more smoothing is required to achieve the partial voluming effect needed for VBM to yield meaningful results.

\section{RESULTS}

\section{Overall findings}

DMl patients showed a significant reduction in TIV (mean (SD) in DMl: 1.380 (0.135); in controls: 1.478 (0.121), $\mathrm{p}=0.005$ ), in WMV (mean (SD) in DMl: 0.390 (0.044); in controls: 0.428 (0.053), p=0.001), and in GMV (mean (SD) in DMl: 0.692 (0.074); in controls: 0.759 (0.065), $p=0.003)$.

Both TIV and GMV inversely correlated with age in patients (TIV: $p<0.02$; GMV: $p=0.000$ ). GMV inversely correlated with age in controls $(p=0.008)$. However, as shown by a significant age $\times$ type (patients $v$ controls) interaction, the inverse correlation between GMV and age was significantly stronger in patients than in controls $(p=0.000)$. WMV did not correlate with age in either patients or controls.

No correlation was observed between brain tissue volumes and grade of disease, disease duration, or CTG expansion. 
The conventional MRI study showed hyperintense lesions in white matter on T2 weighted images (T2LL range: $0.40-$ $8.19 \mathrm{ml}$; median: $1.87 \mathrm{ml}$ ) in all patients. No correlation was observed between brain tissue volumes and T2LL.

\section{Regional grey matter differences}

Local GM volumes were significantly lower in patients than in controls (SPM99 two sample $t$ test, voxel level uncorrected $\mathrm{p}<0.001$, cluster level corrected $\mathrm{p}<0.05$ ) in several cortical areas located in the frontal, parietal, and temporal cortex bilaterally, as well as in the left superior occipital gyrus (table 1 and fig 1). The highest degree of GM atrophy was observed in the bilateral superior parietal lobules, in the left middle and right superior frontal gyrus, and in the right middle and left superior temporal gyrus. Finally, the estimates of subcortical GM showed a significant reduction in the volume of the left caudate.

\section{DISCUSSION}

We used VBM to perform a quantitative analysis of global and regional brain atrophy in $22 \mathrm{DMl}$ patients. Total intracranial volume, WMV, and GMV in DMl patients was significantly lower (from 7 to $9 \%$ ) than in a group of age and sex matched controls, thereby confirming that there is a loss of brain volume in DMl. The cross-sectional design of our study does not allow differentiation between neurodevelopmental volume loss, acquired atrophy, or progressive neurodegeneration; TIV, GMV, and WMV were, however, not found to be influenced either by the duration or severity of disease, though there was a significant age effect on GMV, which was stronger in patients than in controls. This leads us to hypothesise a neurodevelopmental GM loss in the DMl brain, which progresses with ageing. Brain proton MR spectroscopy confirms that the cerebral abnormalities in DMl may be present in an early stage of the disease..$^{13}$ The lack of any correlation between brain atrophy and either CTG expansion or grade of disease suggests that brain involvement in DMl does not progress together with muscular involvement, being linked to different determinants.

The fact that all our patients displayed WM T2 hyperintense lesions which did not correlate with GM loss suggests that WM lesions and cortical atrophy progress as two independent processes.

The analysis of regional GM loss revealed selective regional GM atrophy which, while prevailing in the parietal and frontal lobes, also involved the superior and middle temporal gyrus and occipital lobes. These areas are involved in cognitive dysfunctions and personality disorders, such as deficits in attention, memory, and visuospatial function as well as apathy, depression, and anxiety. Similar symptoms have frequently been reported in DMl, being interpreted as resulting from central nervous system involvement in this disease. $^{914} 15$

VBM may, by detecting atrophic cortical areas and quantifying the degree of atrophy, open an interesting new field of investigation on the relationships between regional cortical atrophy and both intellectual impairment and psychiatric symptoms in DMl.

\section{Authors' affiliations}

G Antonini, F Giubilei, V Ceschin, F Gragnani, S Morino,

A Di Pasquale, Neurological Clinic, II Faculty of Medicine, University of Rome "La Sapienza", Rome, Italy

C Mainero, M Fiorelli, F Caramia, Department of Neurological Sciences, Neuroradiological Section, I Faculty of Medicine, University of Rome "La Sapienza", Rome, Italy

A Romano, Neuroradiological Section, S. Andrea Hospital, Rome, Italy F Soscia, Psychiatric Clinic, II Faculty of Medicine, University of Rome

"La Sapienza", Rome, Italy

Competing interests: none declared

Correspondence to: $\operatorname{Dr} G$ Antonini, Neurological Clinic, S. Andrea Hospital, Via di Grottarossa 1035-1039, 00189 Rome, Italy; giovanni. antonini@uniromal.it

Received 17 November 2003

In revised form 14 February 2004

Accepted 20 February 2004

\section{REFERENCES}

1 Harper PS. Myotonic dystrophy, 3rd ed. Philadelphia, PA: WB Saunders, 2002.

2 Avrahami E, Katz A, Bornstein N, et al. Computed tomographic findings of brain and skull in myotonic dystrophy. J Neurol Neurosurg Psychiatry 1987;50:435-8.

3 Bachmann G, Damian MS, Koch M, et al. The clinical and genetic correlates of MRI findings in myotonic dystrophy. Neuroradiology 1996;38:629-35.

4 Chang L, Anderson T, Migneco OA, et al. Cerebral abnormalities in myotonic dystrophy: cerebral blood flow, magnetic resonance imaging, and neuropsychological tests. Arch Neurol 1993;50:917-23.

5 Fiorelli M, Duboc D, Mazoyer BM, et al. Decreased cerebral glucose utilization in myotonic dystrophy. Neurology 1992;42:91-4.

6 Di Costanzo A, Di Salle F, Santoro L, et al. T2 relaxometry of brain in myotonic dystrophy. Neuroradiology 2001;43:198-204.

7 Naka H, Imon Y, Ohshita T, et al. Magnetization transfer measurements of cerebral white matter in patients with myotonic dystrophy. J Neurol Sci 2002;193:111-6.

8 Kassubek J, Juengling FD, Hoffmann S, et al. Quantification of brain atrophy in patients with myotonic dystrophy and proximal myotonic myopathy: a controlled 3-dimensional magnetic resonance imaging study. Neurosci Lett 2003;348:73-6.

9 Meola G, Sansone V, Perani D, et al. Executive dysfunction and avoidant personality trait in myotonic dystrophy type $1(\mathrm{DM}-1)$ and in proximal myotonic myopathy (PROMM/DM-2). Neuromuscul Disord 2003;13:813-21.

10 Good CD, Johnsrude IS, Ashburner J, et al. A voxel-based morphometric study of ageing in 465 normal adult human brains. Neuroimage $2001 ; 14: 21-36$.

11 Mathieu J, Boivin H, Meunier D, et al. Assessment of a disease-specific muscular impairment rating scale in myotonic dystrophy. Neurology $2001 ; 56: 336-40$.

12 Friston KJ, Holmes AP, Worsley KJ, et al. Statistical parametric maps in functional imaging: a general linear approach. Hum Brain Mapp 1995;2:189-210.

13 Akiguchi I, Nakano S, Shiino A, et al. Brain proton magnetic resonance spectroscopy and brain atrophy in myotonic dystrophy. Arch Neurol 1999;56:325-30.

14 Abe K, Fujimura H, Toyooka K, et al. Involvement of the central nervous system in myotonic dystrophy. J Neurol Sci 1994; 127:179-85.

15 Ashizawa T. Myotonic dystrophy as a brain disorder. Arch Neurol 1998:55:291-3. 